\title{
The occipitofrontal circumference: reliable prediction of the intracranial volume in children with syndromic and complex craniosynostosis
}

\author{
*Bianca Francisca Maria Rijken, MD,' Bianca Kelly den Ottelander, BSc, \\ Marie-Lise Charlotte van Veelen, MD, ${ }^{3}$ Maarten Hans Lequin, MD, $\mathrm{PhD},{ }^{2}$ and \\ Irene Margreet Jacqueline Mathijssen, MD, PhD'
}

1Department of Plastic and Reconstructive Surgery, Craniofacial Center, ${ }^{2}$ Department of Pediatric Radiology, and ${ }^{3}$ Department of Pediatric Neurosurgery, Erasmus University Medical Center-Sophia Children's Hospital, Rotterdam, The Netherlands

\begin{abstract}
OBJECT Patients with syndromic and complex craniosynostosis are characterized by the premature fusion of one or more cranial sutures. These patients are at risk for developing elevated intracranial pressure (ICP). There are several factors known to contribute to elevated ICP in these patients, including craniocerebral disproportion, hydrocephalus, venous hypertension, and obstructive sleep apnea. However, the causal mechanism is unknown, and patients develop elevated ICP even after skull surgery. In clinical practice, the occipitofrontal circumference (OFC) is used as an indirect measure for intracranial volume (ICV), to evaluate skull growth. However, it remains unknown whether OFC is a reliable predictor of ICV in patients with a severe skull deformity. Therefore, in this study the authors evaluated the relation between ICV and OFC.
\end{abstract}

METHODS Eighty-four CT scans obtained in 69 patients with syndromic and complex craniosynostosis treated at the Erasmus University Medical Center-Sophia Children's Hospital were included. The ICV was calculated based on CT scans by using autosegmentation with an HU threshold < 150. The OFC was collected from electronic patient files. The CT scans and OFC measurements were matched based on a maximum amount of the time that was allowed between these examinations, which was dependent on age. A Pearson correlation coefficient was calculated to evaluate the correlations between OFC and ICV. The predictive value of OFC, age, and sex on ICV was then further evaluated using a univariate linear mixed model. The significant factors in the univariate analysis were subsequently entered in a multivariate mixed model.

RESULTS The correlations found between OFC and ICV were $r=0.908$ for the total group $(p<0.001), r=0.981$ for Apert $(p<0.001), r=0.867$ for Crouzon-Pfeiffer $(p<0.001), r=0.989$ for Muenke $(p<0.001), r=0.858$ for SaethreChotzen syndrome $(p=0.001)$, and $r=0.917$ for complex craniosynostosis $(p<0.001)$. Age and OFC were significant predictors of ICV in the univariate linear mixed model ( $p<0.001$ for both factors). The OFC was the only predictor that remained significant in the multivariate analysis $(p<0.001)$.

CONCLUSIONS The OFC is a significant predictor of ICV in patients with syndromic and complex craniosynostosis. Therefore, measuring the OFC during clinical practice is very useful in determining which patients are at risk for impaired skull growth.

http://thejns.org/doi/abs/10.3171/2015.2.FOCUS14846

KEY WORDS syndromic craniosynostosis; occipitofrontal circumference; intracranial volume

$\mathrm{C}$ RANIOSYNOSTOSIS involves the premature fusion of one or more cranial sutures, which results in deformation of the skull due to lack of growth perpendicular to the affected suture and compensatory overgrowth at the nonaffected sutures. ${ }^{17}$ This rare condition, with a prevalence of 1 in $2100-2500$ births, ${ }^{17}$ is classified as syndromic in up to $24 \%$ of cases.$^{27}$ Syndromes associated with craniosynostosis include Apert, Crouzon-Pfeiffer, Muenke, and Saethre-Chotzen. ${ }^{17}$ Patients in whom more than one suture is affected, but for which no responsible gene mutation has been found, are called complex cases. ${ }^{18,19}$

ABBREVIATIONS HU = Hounsfield unit; ICP = intracranial pressure; ICV = intracranial volume; OFC = occipitofrontal circumference.

SUBMITTED December 27, 2014. ACCEPTED February 12, 2015 INCLUDE WHEN CITING DOI: 10.3171/2015.2.FOCUS14846.

DISCLOSURE The authors report no conflict of interest concerning the materials or methods used in this study or the findings specified in this paper.

* Dr. Rijken and Mrs. den Ottelander contributed equally to this work. 
The treatment of craniosynostosis consists of skull vault surgery. This procedure is performed to prevent or to treat elevated intracranial pressure (ICP) by enlarging the intracranial volume (ICV). Surgery is preferably performed within 1 year after birth, because the risk of developing raised ICP is higher when the operation is performed later in life. ${ }^{24}$ Furthermore, the mental outcome is better compared with patients who undergo a cranial vault expansion later in life. ${ }^{24}$ Moreover, the skull deformity is corrected and its progression is prevented. ${ }^{17}$ In general, patients with Apert and Crouzon-Pfeiffer syndromes underwent an occipital vault expansion, which might be followed by a frontoorbital advancement later in life, whereas patients with Muenke and Saethre-Chotzen syndromes underwent a frontoorbital advancement as the first (and often the only) surgical procedure. For patients with multisuture synostosis, the surgical procedure depends on the cranial sutures involved; occipital expansion when lambdoid sutures are involved, and frontoorbital advancement when coronal sutures are involved.

Preoperatively, $40 \%-50 \%$ of the patients with Apert syndrome, $50 \%-70 \%$ of those with Crouzon-Pfeiffer syndrome, $35 \%-45 \%$ of those with Saethre-Chotzen syndrome, $50 \%-80 \%$ of the patients with complex craniosynostosis, and none of those with Muenke syndrome develop increased ICP. . $12,13,15,18,21,24,25,29,30,32$ Postoperatively, $35 \%-43 \%$ of patients still develop raised ICP. ${ }^{12,13,15,18,25,29,30}$ Factors influencing ICP in these patients include craniocerebral disproportion (a condition in which the brain grows faster than the skull), hydrocephalus, venous hypertension, and obstructive sleep apnea syndrome. . $^{8,11,14}$

During follow-up of the surgically treated patient, the occipitofrontal circumference (OFC) is used as a derivative of ICV to evaluate whether craniocerebral disproportion might be present. However, it is unknown whether OFC and ICV correlate in patients with a severe skull deformity. Although OFC and ICV have been shown to correlate in healthy individuals, ${ }^{6,7}$ a small study $(n=7)$ in infants with an abnormal head shape, including 5 with craniosynostosis, could not find any correlation. ${ }^{7}$ The aim of the present study is to evaluate the correlation between OFC and ICV in children with different craniosynostosis syndromes, and to assess the predictive value of OFC in these patients.

\section{Methods \\ Data Acquisition}

The volume measurements were performed using 3D CT scans. These scans were assembled via the radiology department of the Erasmus University Medical CenterSophia Children's Hospital. All digitally available 3D CT scans of the skull (slice thickness $1.25-3 \mathrm{~mm}$ ) in all children (age 0-18 years) with syndromic and complex craniosynostosis that were obtained between January 2000 and January 2014 were initially included. The exclusion criteria were as follows: incomplete scans (scans that did not totally include the area between the vertex and the foramen magnum), and scans with artifacts caused by the presence of distractors. Scans including contrast media were also excluded from the study, due to the highly timeconsuming measurement.
Data on OFC were collected from electronic patient files. The 3D CT scans and OFCs were paired based on the time interval between the CT scan and OFC measurement; because the human skull grows mostly in the first months of life, ${ }^{10}$ the OFC had to be measured within 1 month before or after the CT scan for children $0-1$ years, within 3 months for patients 1-2 years, within 6 months for those 2-4 years of age, and 12 months for those $>4$ years (Table 1). Additionally, the OFC and CT scan were not paired when the patient underwent skull surgery between the measurement of OFC and acquisition of the CT scan.

\section{The ICV Measurement}

The ICV was calculated from 3D CT scans by using Brainlab, a neuronavigation program. Within this software, autosegmentation with a soft-tissue/bone Hounsfield unit (HU) threshold of $<150 \mathrm{HU}^{1-4}$ was performed to outline the ICV. Parts with an HU value below 150, such as skin, that did not contribute to the ICV were manually excluded using axial, sagittal, and coronal images (Fig. 1). These planes were also used to outline the foramen magnum manually, using the area between the clivus and the occipital bone as a cutoff point in the sagittal plane (Fig. 2). When the outlining was finished on each slice, the software program automatically calculated the ICV (Fig. 3). The intrarater reliability was based on the intraclass correlation coefficient, which was 1.000 .

\section{Statistical Analysis}

To study the correlation between OFC and ICV, a Pearson correlation coefficient was calculated for all patients combined, and per syndrome. Additionally, correlations were calculated as follows: 1) preoperatively; 2) postoperatively; 3) for Apert syndrome patients with a turricephaly; or 4) those without a turricephaly. Only the first 3D CT scan for each patient was used for the calculation of the correlations, because correlation coefficients do not allow the use of multiple measurements. For further analysis of the predictive value of OFC, a linear mixed model correcting for multiple measurements, age, and sex was used. The OFC, age, and sex were first entered separately into the model. Variables that were significant in the univariate analysis were subsequently entered into a multivariate linear mixed model. Statistical significance was defined as $\mathrm{p}<0.05$.

\section{Results}

One hundred fifty-nine of 229 CT scans remained eligible when the inclusion and exclusion criteria were

TABLE 1. Matching criteria for CT scans and OFC measurements

\begin{tabular}{cc}
\hline Age $(\mathrm{yrs})$ & ${\text { Interval Allowed }(\mathrm{mos})^{*}}^{*}$ \\
\hline $0-1$ & 1 \\
\hline $1-2$ & 3 \\
\hline $2-4$ & 6 \\
\hline$>4$ & 12 \\
\hline
\end{tabular}

* The time interval between CT scans and OFC measurements. 

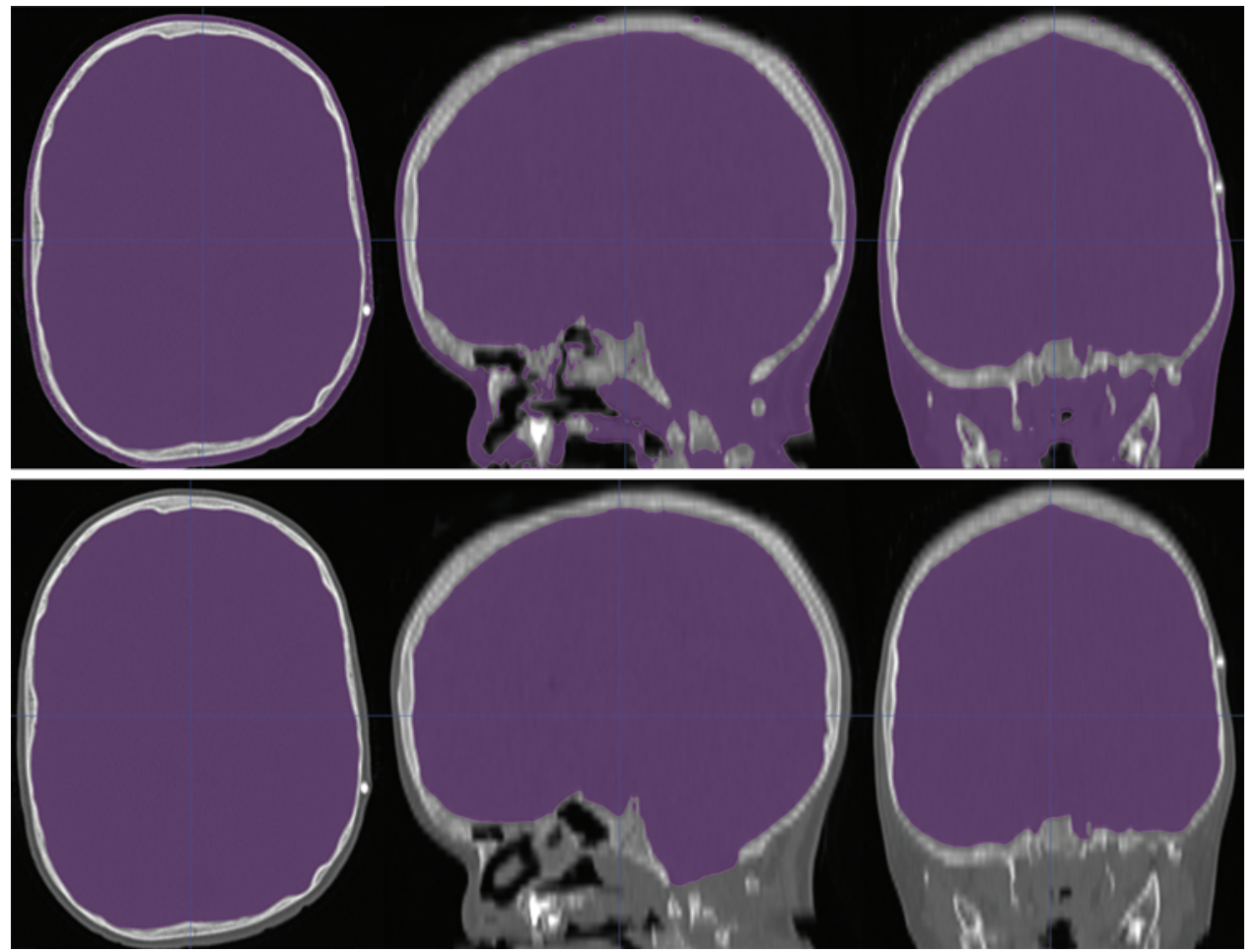

FIG. 1. Upper Row: Axial, sagittal, and coronal 3D CT scans showing results after autosegmentation, which includes all tissue with an $\mathrm{HU}$ value < 150. This excludes bone, but includes brain, eyes, skin, mucosa, and other soft tissue (for example, that of the nasopharyngeal area). Lower Row: Axial, sagittal, and coronal 3D CT scans showing results after manual correction, whereby soft tissues other than brain, ventricles containing CSF, and blood vessels containing blood were manually erased. Consequently, only the ICV remained, of which the lower border was defined by the foramen magnum.

applied, of which 84 were paired with an OFC measurement (Fig. 4). These 84 scans were obtained in 69 patients with craniosynostosis, including Apert $(\mathrm{n}=11)$, CrouzonPfeiffer $(n=24)$, Muenke $(n=7)$, and Saethre-Chotzen ( $=10)$ syndromes, and complex craniosynostosis $(n=17)$

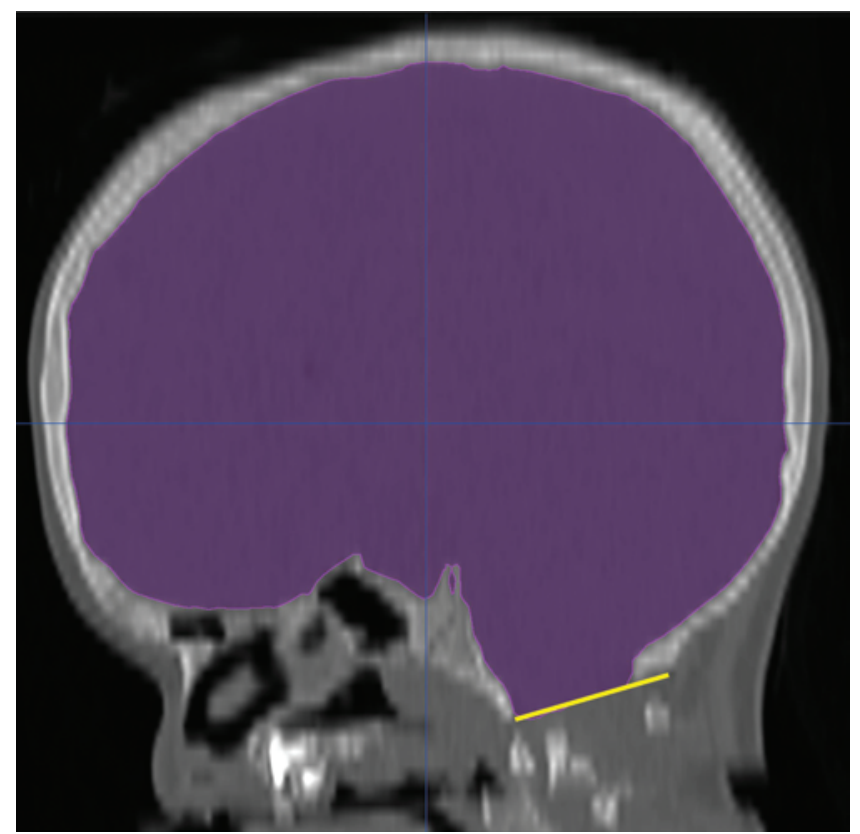

FIG. 2. A 3D CT scan on which the foramen magnum between the clivus and occipital bone is outlined (yellow line).
(Table 2). Three patients with Apert, 6 with Crouzon-Pfeiffer, and 1 with Saethre-Chotzen syndrome, and 1 with complex craniosynostosis underwent multiple scans. The mean age of our patient population was 5.7 years (range

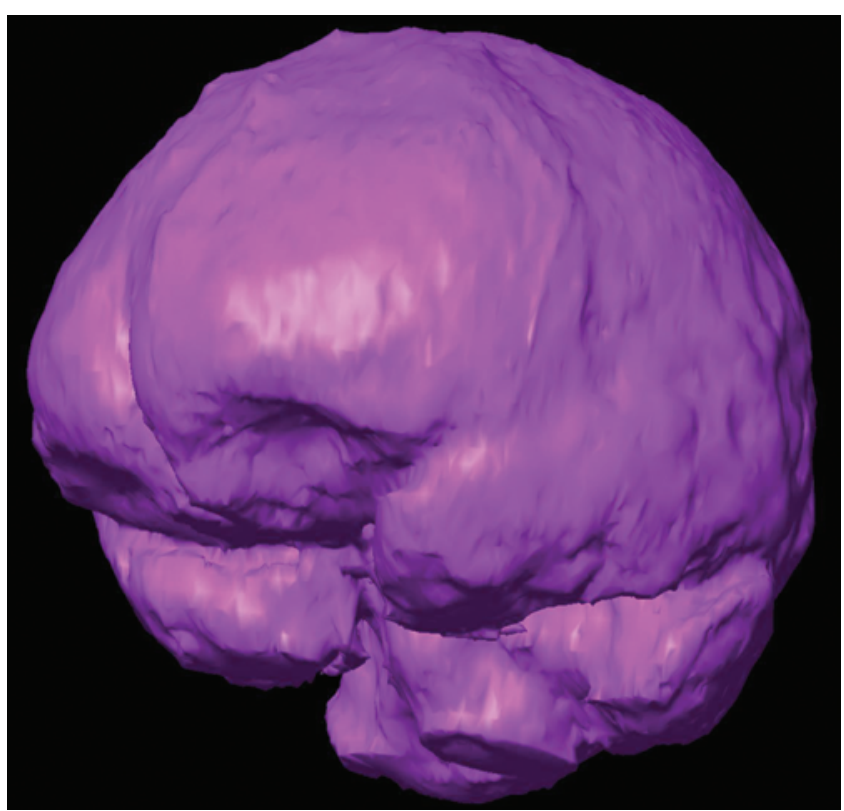

FIG. 3. A 3D reconstruction of the ICV, after manually correcting the automatic measurement. This included the brain, ventricles with CSF cerebellum, upper part of the brainstem (above the foramen magnum), and blood vessels. 


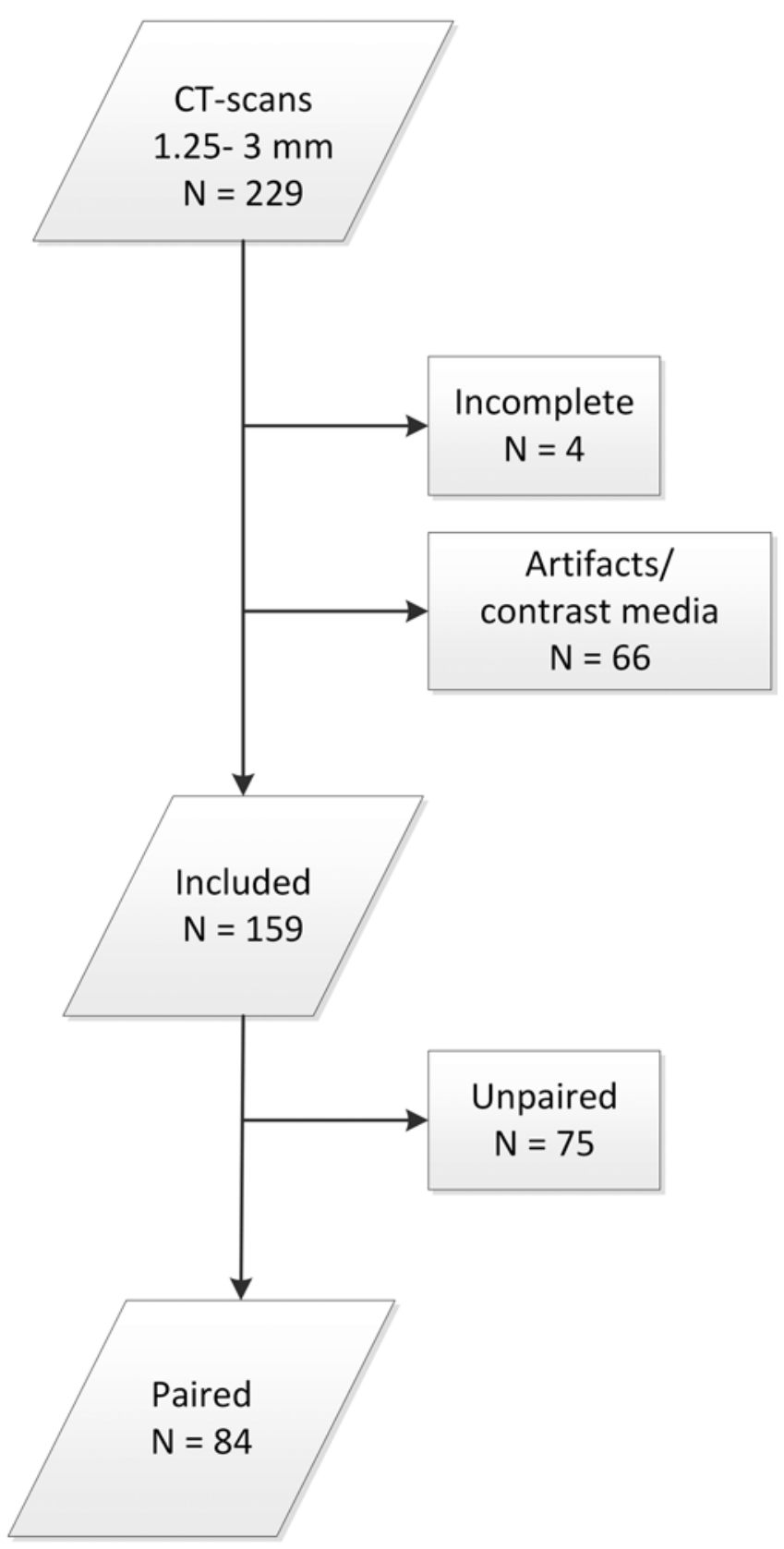

FIG. 4. Flowchart of the scans included in the study.

2 months to 18 years), and 34 of the patients were female (49\%).

The correlation coefficient between OFC and ICV was $r=0.908$ for all patients combined; $r=0.981$ for those with Apert; $r=0.867$ for those with Crouzon-Pfeiffer; $r=0.989$ for those with Muenke; $r=0.858$ for those with SaethreChotzen syndrome; and $\mathrm{r}=0.917$ for those with complex craniosynostosis (Tables 3 and 4). Preoperatively, the correlation between OFC and ICV for all patients combined was $r=0.903(p<0.001)$, and it was $r=0.815$ postoperatively $(\mathrm{p}<0.001)$. The correlation in patients with Apert syndrome with a turricephaly $(n=4)$ was $r=0.984(p<$ 0.031 ), and for patients with Apert syndrome without a turricephaly $(\mathrm{n}=7)$ it was $\mathrm{r}=0.969(\mathrm{p}<0.001)$. In the univariate analysis, OFC and age were significant predictors
TABLE 2. Characteristics of 69 patients with syndromic and complex craniosynostosis

\begin{tabular}{lcc}
\hline \multicolumn{1}{c}{ Characteristic } & No. of Patients $(\%)$ & No. of Scans $(\%)$ \\
\hline Total & 69 & 84 \\
\hline Craniosynostosis group & & \\
\hline Apert & $11(16)$ & $17(20)$ \\
\hline Crouzon-Pfeiffer & $24(35)$ & $31(37)$ \\
\hline Muenke & $7(10)$ & $7(8)$ \\
\hline Saethre-Chotzen & $10(14)$ & $11(13)$ \\
\hline Complex & $17(25)$ & $18(21)$ \\
\hline Sex & & \\
\hline Male & $35(51)$ & $44(52)$ \\
\hline Female & $34(49)$ & $40(48)$ \\
\hline Age in yrs & & $25(30)$ \\
\hline $0-1$ & $25(36)$ & $9(11)$ \\
\hline $1-2$ & $8(12)$ & $15(18)$ \\
\hline $2-4$ & $10(14)$ & $7(8)$ \\
\hline $4-8$ & $6(9)$ & $10(12)$ \\
\hline $8-12$ & $9(13)$ & $18(21)$ \\
\hline $12-18$ & $11(16)$ &
\end{tabular}

of ICV ( $p<0.001$ for both factors), whereas sex had no significant contribution $(\mathrm{p}=0.238)$. When OFC and age were entered into a multivariate model, OFC remained the only significant predictor of the ICV ( $p<0.001$ for OFC and $\mathrm{p}=0.136$ for age).

\section{Discussion}

In this study we evaluated the predictive value of OFC for ICV, and the correlation between OFC and ICV in patients with syndromic and complex craniosynostosis. The OFC and ICV were highly correlated when evaluating all patients combined, but also for subgroups per syndrome, pre- and postoperatively, and in patients with Apert syndrome with a turricephaly. Furthermore, when corrected for age, OFC was a significant predictor of ICV. The OFC and ICV were highly correlated in our study population.

A previous study in which the correlation between OFC and ICV was evaluated could not find any correlation.? However, we believe that our study has several strengths in comparison with the previous one. For example, the study population of Buda et al. consisted of only 7 infants with a deviated skull form, of whom 5 patients had craniosynos-

TABLE 3. The mean ICV, OFC, and age per craniosynostosis group

\begin{tabular}{lcccc}
\hline \multicolumn{1}{c}{ Group } & $\begin{array}{c}\text { Age at } \\
\text { CT (yrs) }\end{array}$ & ICV $\left(\mathrm{cm}^{3}\right)$ & $\begin{array}{c}\text { Age at } \\
\text { OFC }(\mathrm{yrs})\end{array}$ & OFC $(\mathrm{cm})$ \\
\hline Total & 5.7 & 1242 & 5.7 & 48.3 \\
\hline Apert & 8.5 & 1590 & 8.5 & 50.6 \\
\hline Crouzon-Pfeiffer & 8.2 & 1357 & 8.2 & 51.6 \\
\hline Muenke & 3.1 & 1082 & 3.2 & 45.2 \\
\hline Saethre-Chotzen & 3.1 & 1070 & 3.0 & 45.7 \\
\hline Complex & 1.5 & 884 & 1.5 & 43.1 \\
\hline
\end{tabular}


TABLE 4. Correlations between OFC and ICV per craniosynostosis group

\begin{tabular}{ccc}
\hline Group & Correlation & $p$ Value \\
\hline Total & 0.908 & $<0.001$ \\
\hline Apert & 0.981 & $<0.001$ \\
\hline Crouzon-Pfeiffer & 0.867 & $<0.001$ \\
\hline Muenke & 0.989 & $<0.001$ \\
\hline Saethre-Chotzen & 0.858 & 0.001 \\
\hline Complex & 0.917 & $<0.001$ \\
\hline
\end{tabular}

tosis, whereas our study consisted of a much larger cohort. Additionally, ICVs in the previous study were calculated using radiographs, whereas in this study we calculated the ICV on 3D CT scans, which is a more established method for ICV measurements. ${ }^{26}$ There are 2 studies in which the correlation between OFC and ICV was evaluated in healthy individuals. ${ }^{6,7}$ They found correlations of $r=0.97$ and $r=0.98$, which is slightly higher than the correlation of $r=0.91$ that we found in our study population. Apparently, although the skull shape is altered in children with craniosynostosis, it turns out that the OFC is still a reliable tool to evaluate ICV.

For each syndrome separately, OFC and ICV were highly correlated as well. We believed that the correlation might be altered in children with Apert syndrome, due to the presence of patients with a turricephaly in this group. However, we found that the correlation in Apert syndrome was one of the highest of all syndrome subgroups, and additionally that the presence or absence of a turricephaly did not alter the correlation substantially. Whether OFC and ICV have a good correlation in patients with CrouzonPfeiffer syndrome with only sagittal suture synostosis was evaluated in 2 patients. Remarkably, the correlation coefficient of these 2 patients was almost identical to the rest of the group ( 0.867 and 0.865 , respectively). However, this might be explained by the fact that both patients were measured postoperatively, indicating that the configuration of the skull in these patients was altered, and was more similar to those without sagittal suture involvement. Therefore, it is uncertain whether the OFC is as good a predictor for ICV in patients with Crouzon-Pfeiffer syndrome with only sagittal suture synostosis prior to correction.

In addition, ICV depends on the diagnosis; ICV is normal in unicoronal suture synostosis,${ }^{16}$ normal or slightly enlarged in sagittal suture synostosis, ${ }^{20,23}$ and enlarged in patients with Apert and Crouzon-Pfeiffer syndromes. ${ }^{22}$ Increasing ICV is caused by increasing brain mass or CSF, which both might contribute to elevated ICP. The incidence of increased ICP differs between different craniosynostosis syndromes, with the highest percentage in patients with Crouzon-Pfeiffer syndrome. ${ }^{5}$ In addition, patients with different craniosynostosis syndromes show different OFC curves, and some are regarded as more likely to develop a deflecting OFC growth curve than others. ${ }^{28}$ For example, stagnating skull growth is more often seen in patients with Crouzon-Pfeiffer syndrome than in patients with Muenke syndrome. Therefore we studied the OFC curves per syndrome, and investigated the relation between OFC and ICV per syndrome as well. For each craniosynostosis syndrome, the relation between ICV and OFC was high, which means that the OFC curve is a reliable measurement to study ICV in different craniosynostosis syndromes. This makes it a clinically useful tool to study skull growth. Moreover, it turns out that a deflecting growth curve (OFC not changing or showing growth of $<0.5 \mathrm{SD}$ within 2 years) is a very important risk factor for developing papilledema, which is an indirect sign of increased ICP. $9,28,31$

A limitation of our study includes its retrospective design. The OFC was often not measured on the day that the CT scan was performed. To evaluate the influence of our variables more precisely, these measurements would preferably be made on the same day. Another point of attention might be the human factor when measuring the OFC; for example, measurement errors or noncooperative children. However, the data collection was done by experienced professionals at every visit of the patient to the clinic, which limits those factors as much as possible.

\section{Conclusions}

Taken together, craniocerebral disproportion contributes to the development of elevated ICP in children with syndromic and complex craniosynostosis. The ICV measurements can be used to monitor skull growth. However, because this is time consuming and therefore not feasible for use in clinical practice, OFC is often used as an alternative to ICV. We found that $\mathrm{OFC}$ is indeed a reliable predictor of ICV in the total group of patients with craniosynostosis, in the individual syndromes as well as in surgically and nonsurgically treated patients with craniosynostosis. This makes it a rapid and accurate method to monitor skull growth during follow-up and to help determine which patients are lacking in ICV.

\section{References}

1. Abbott AH, Netherway DJ, Niemann DB, Clark B, Yamamoto M, Cole J, et al: CT-determined intracranial volume for a normal population. J Craniofac Surg 11:211-223, 2000

2. Anderson PJ, Netherway DJ, Abbott A, David DJ: Intracranial volume measurement of metopic craniosynostosis. J Craniofac Surg 15:1014-1018, 2004

3. Anderson PJ, Netherway DJ, Abbott AH, Cox T, Roscioli T, David DJ: Analysis of intracranial volume in apert syndrome genotypes. Pediatr Neurosurg 40:161-164, 2004

4. Anderson PJ, Netherway DJ, McGlaughlin K, David DJ: Intracranial volume measurement of sagittal craniosynostosis. J Clin Neurosci 14:455-458, 2007

5. Bannink N, Joosten KF, van Veelen ML, Bartels MC, Tasker $\mathrm{RC}$, van Adrichem LN, et al: Papilledema in patients with Apert, Crouzon, and Pfeiffer syndrome: prevalence, efficacy of treatment, and risk factors. J Craniofac Surg 19:121-127, 2008

6. Bray PF, Shields WD, Wolcott GJ, Madsen JA: Occipitofrontal head circumference - an accurate measure of intracranial volume. J Pediatr 75:303-305, 1969

7. Buda FB, Reed JC, Rabe EF: Skull volume in infants. Methodology, normal values, and application. Am J Dis Child 129:1171-1174, 1975

8. Collmann H, Sörensen N, Krauss J: Hydrocephalus in craniosynostosis: a review. Childs Nerv Syst 21:902-912, 2005

9. Connolly JP, Gruss J, Seto ML, Whelan MF, Ellenbogen 
$\mathrm{R}$, Weiss A, et al: Progressive postnatal craniosynostosis and increased intracranial pressure. Plast Reconstr Surg 113:1313-1323, 2004

10. de Jong T, Rijken BF, Lequin MH, van Veelen ML, Mathijssen IM: Brain and ventricular volume in patients with syndromic and complex craniosynostosis. Childs Nerv Syst 28:137-140, 2012

11. Driessen C, Joosten KF, Bannink N, Bredero-Boelhouwer HH, Hoeve HL, Wolvius EB, et al: How does obstructive sleep apnoea evolve in syndromic craniosynostosis? A prospective cohort study. Arch Dis Child 98:538-543, 2013

12. Gault DT, Renier D, Marchac D, Jones BM: Intracranial pressure and intracranial volume in children with craniosynostosis. Plast Reconstr Surg 90:377-381, 1992

13. Greene AK, Mulliken JB, Proctor MR, Meara JG, Rogers GF: Phenotypically unusual combined craniosynostoses: presentation and management. Plast Reconstr Surg 122:853862,2008

14. Hayward R: Venous hypertension and craniosynostosis. Childs Nerv Syst 21:880-888, 2005

15. Hayward R, Gonsalez S: How low can you go? Intracranial pressure, cerebral perfusion pressure, and respiratory obstruction in children with complex craniosynostosis. J Neurosurg 102 (1 Suppl):16-22, 2005

16. Hill CA, Vaddi S, Moffitt A, Kane AA, Marsh JL, Panchal J, et al: Intracranial volume and whole brain volume in infants with unicoronal craniosynostosis. Cleft Palate Craniofac J 48:394-398, 2011

17. Johnson D, Wilkie AO: Craniosynostosis. Eur J Hum Genet 19:369-376, 2011

18. Kress W, Schropp C, Lieb G, Petersen B, Büsse-Ratzka M, Kunz J, et al: Saethre-Chotzen syndrome caused by TWIST 1 gene mutations: functional differentiation from Muenke coronal synostosis syndrome. Eur J Hum Genet 14:39-48, 2006

19. Lajeunie E, Heuertz S, El Ghouzzi V, Martinovic J, Renier $\mathrm{D}$, Le Merrer M, et al: Mutation screening in patients with syndromic craniosynostoses indicates that a limited number of recurrent FGFR2 mutations accounts for severe forms of Pfeiffer syndrome. Eur J Hum Genet 14:289-298, 2006

20. Lee SS, Duncan CC, Knoll BI, Persing JA: Intracranial compartment volume changes in sagittal craniosynostosis patients: influence of comprehensive cranioplasty. Plast Reconstr Surg 126:187-196, 2010

21. Marucci DD, Dunaway DJ, Jones BM, Hayward RD: Raised intracranial pressure in Apert syndrome. Plast Reconstr Surg 122:1162-1170, 2008

22. Posnick JC, Armstrong D, Bite U: Crouzon and Apert syndromes: intracranial volume measurements before and after cranio-orbital reshaping in childhood. Plast Reconstr Surg 96:539-548, 1995

23. Posnick JC, Armstrong D, Bite U: Metopic and sagittal synostosis: intracranial volume measurements prior to and after cranio-orbital reshaping in childhood. Plast Reconstr Surg 96:299-315, 1995
24. Renier D, Lajeunie E, Arnaud E, Marchac D: Management of craniosynostoses. Childs Nerv Syst 16:645-658, 2000

25. Renier D, Sainte-Rose C, Marchac D, Hirsch JF: Intracranial pressure in craniostenosis. J Neurosurg 57:370-377, 1982

26. Sahin B, Mazonakis M, Akan H, Kaplan S, Bek Y: Dependence of computed tomography volume measurements upon section thickness: an application to human dry skulls. Clin Anat 21:479-485, 2008

27. Sharma VP, Fenwick AL, Brockop MS, McGowan SJ, Goos JA, Hoogeboom AJ, et al: Mutations in TCF12, encoding a basic helix-loop-helix partner of TWIST1, are a frequent cause of coronal craniosynostosis. Nat Genet 45:304-307, 2013

28. Spruijt B, Joosten KFM, Driessen C, Rizopoulos D, Naus NC, van der Schroeff MP, et al: Algorithm for the management of intracranial hypertension in children with syndromic craniosynostosis. Plast Reconstr Surg [in press], 2015

29. Tamburrini G, Di Rocco C, Velardi F, Santini P: Prolonged intracranial pressure (ICP) monitoring in non-traumatic pediatric neurosurgical diseases. Med Sci Monit 10:MT53MT63, 2004

30. Thompson DN, Harkness W, Jones B, Gonsalez S, Andar U, Hayward R: Subdural intracranial pressure monitoring in craniosynostosis: its role in surgical management. Childs Nerv Syst 11:269-275, 1995

31. Tuite GF, Chong WK, Evanson J, Narita A, Taylor D, Harkness WF, et al: The effectiveness of papilledema as an indicator of raised intracranial pressure in children with craniosynostosis. Neurosurgery 38:272-278, 1996

32. Woods RH, Ul-Haq E, Wilkie AO, Jayamohan J, Richards PG, Johnson D, et al: Reoperation for intracranial hypertension in TWIST1-confirmed Saethre-Chotzen syndrome: a 15-year review. Plast Reconstr Surg 123:1801-1810, 2009

\section{Author Contributions}

Conception and design: Rijken, van Veelen, Mathijssen. Acquisition of data: den Ottelander, Lequin, Mathijssen. Analysis and interpretation of data: Rijken, den Ottelander, van Veelen, Mathijssen. Drafting the article: all authors. Critically revising the article: all authors. Reviewed submitted version of manuscript: all authors. Approved the final version of the manuscript on behalf of all authors: Rijken. Statistical analysis: den Ottelander. Administrative/technical/material support: Rijken, Lequin. Study supervision: Rijken, Lequin, Mathijssen.

\section{Correspondence}

Bianca Francisca Maria Rijken, Department of Plastic, Reconstructive, and Hand Surgery, Rm. Ee 15.91, Erasmus University Medical Center, P.O. Box 2040, 3000 CA Rotterdam, The Netherlands.email: b.rijken@erasmusmc.nl. 